\title{
Representaciones sociales de la gestión ambiental para la sustentabilidad en una comunidad escolar privada
}

\author{
Social Representations of Environmental Management for Sustainability in \\ a Private School Community
} \begin{abstract}
Representações sociais da gestão ambiental para a sustentabilidade em
\end{abstract} uma comunidade escolar privada

Trinidad Esmeralda Vilchis Pérez Universidad Autónoma de Guerrero, México tvilchis@uagro.mx https://orcid.org/0000-0003-4394-5277

José Luis Aparicio López Universidad Autónoma de Guerrero, México joselopez@uagro.mx https://orcid.org/0000-0002-4586-6954

Esperanza Terrón Amigón Universidad Pedagógica Nacional, México eterron@upn.mx https://orcid.org/0000-0001-9707-1953

Columba Rodríguez Alviso Universidad Autónoma de Guerrero, México columbaalviso@uagro.mx https://orcid.org/0000-0001-9600-8776

Hilda Janet Arellano Wences Universidad Autónoma de Guerrero, México hjarellano@uagro.mx https://orcid.org/0000-0001-7308-7731 


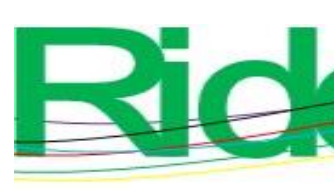

Revista Iberoamericana para la Investigación y el Desarrollo Educativo ISSN $2007-7467$

\section{Resumen}

El objetivo de esta investigación fue identificar las representaciones sociales sobre la gestión ambiental para la sustentabilidad compartidas por la comunidad escolar de la Universidad Español campus Diamante en Acapulco, Guerrero. El alcance metodológico fue descriptivo y el diseño mixto. Se aplicaron 276 cuestionarios y 11 entrevistas al personal directivo, docente y no académico y a estudiantes. Los datos cualitativos se procesaron mediante análisis de contenido y los cuantitativos a través de redes semánticas naturales y estadística descriptiva. En cuanto al cuestionario, las respuestas predominantes indicaron que la información adquirida por la comunidad escolar proviene, principalmente, de asignaturas como la de biología (26.7\%); en relación con las representaciones sociales, $41 \%$ de los entrevistados piensa que el concepto gestión ambiental se enfoca al cuidado, $31 \%$ desconoce la sustentabilidad y $30 \%$ relaciona la sustentabilidad con la conservación de los recursos naturales. Asimismo, para $47 \%$ de los encuestados, la finalidad de la gestión ambiental es, básicamente, ahorrar y administrar los recursos. Se concluye con la necesidad de desarrollar procesos de formación con toda la comunidad escolar para transitar de una conceptualización conservacionista y utilitarista a la construcción de representaciones sociales más sólidas, complejas y dinámicas, orientadas a la sustentabilidad.

Palabras clave: educación ambiental, medio ambiente, organización y gestión, percepción.

\section{Abstract}

The objective of this research was to identify the social representations about environmental management for sustainability shared by the school community of Universidad Español Diamante campus in Acapulco, Guerrero. The methodological scope was descriptive, and the design was mixed; 276 questionnaires and 11 interviews were applied to directive, teaching and non-academic staff and students. Qualitative data were processed through content analysis and quantitative data through natural semantic networks and descriptive statistics. Regarding the questionnaire, predominant responses indicated that the information acquired by the school community comes mainly from subjects taken in their academic training (biology, $26.7 \%$ ); In relation to social representations, $41 \%$ of those interviewed think that the concept environmental management focuses on care, $31 \%$ are unaware of 


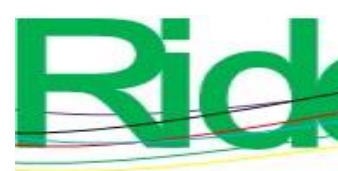

Revista Iberoamericana para la Investigación y el Desarrollo Educativo ISSN $2007-7467$

sustainability and $30 \%$ relate sustainability to the conservation of natural resources. Likewise, for $47 \%$ of those surveyed, the purpose of environmental management is basically to save and manage resources. The need to develop training processes with the entire school community is concluded, to move from a conservationist and utilitarian conceptualization to the construction of more solid, complex, and dynamic social representations, oriented towards sustainability.

Keywords: environmental education, environment, organization and management, perception.

\section{Resumo}

O objetivo desta pesquisa foi identificar as representações sociais sobre a gestão ambiental para a sustentabilidade compartilhadas pela comunidade escolar do campus da Universidad Español Diamante em Acapulco, Guerrero. O escopo metodológico foi descritivo e o desenho misto. Foram aplicados 276 questionários e 11 entrevistas a funcionários directivos, docentes e não académicos e alunos. Os dados qualitativos foram processados por meio da análise de conteúdo e os dados quantitativos por meio de redes semânticas naturais e estatísticas descritivas. Em relação ao questionário, as respostas predominantes indicaram que as informações adquiridas pela comunidade escolar provêm principalmente de disciplinas como biologia $(26,7 \%)$; Em relação às representações sociais, $41 \%$ dos entrevistados acham que o conceito de gestão ambiental tem como foco o cuidado, $31 \%$ desconhecem a sustentabilidade e $30 \%$ relacionam a sustentabilidade à conservação dos recursos naturais. Da mesma forma, para $47 \%$ dos pesquisados, a finalidade da gestão ambiental é basicamente economizar e administrar recursos. Conclui com a necessidade de desenvolver processos de formação com toda a comunidade escolar para passar de uma conceituação conservacionista e utilitária à construção de representações sociais mais sólidas, complexas e dinâmicas, orientadas para a sustentabilidade.

Palavras-chave: educação ambiental, meio ambiente, organização e gestão, percepção. Fecha Recepción: Junio 2021

Fecha Aceptación: Noviembre 2021 


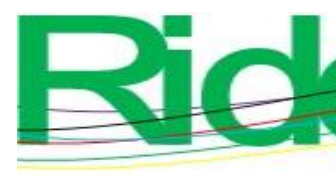

Revista Iberoamericana para la Investigación y el Desarrollo Educativo ISSN 2007-7467

\section{Introducción}

Los sistemas de gestión ambiental surgen como una estrategia para contribuir a la identificación y control de los impactos que las organizaciones generan en el ambiente (Malavé y Fernández, 2020; Organización Internacional de Normalización [ISO, por sus siglas en inglés] (2015). Las investigaciones iniciales en este campo se enfocaron en el ámbito empresarial con el fin de valorar la eficacia de la implementación de la norma ISO 14001 (Mikulčić, Duić y Dewil, 2017; Salim et al., 2017), la importancia del manejo de residuos sólidos (So, Lee y Chow, 2019) u otros aspectos, como la relación entre gestión ambiental, desempeño económico, innovación verde y liderazgo (Zhang y Ma, 2021). Actualmente se abordan tópicos como el comportamiento, la participación y el compromiso para adoptar prácticas de gestión ambiental (Kitila y Woldemikael, 2019; Klein, Schramm, Veiga y Moreira, 2021; León et al., 2017; Mills et al., 2017).

No obstante, las diversas manifestaciones de la crisis ambiental hicieron evidente la necesidad de fomentar la participación de un mayor número de personas en la búsqueda de soluciones para una convivencia armónica entre sociedad y naturaleza. La Declaración de Talloires (Association of University Leaders for a Sustainable Future, 1999) reconoce que las instituciones de educación superior juegan un papel fundamental en el desarrollo de estrategias de sustentabilidad para atender la problemática actual. Una de ellas es la incorporación de sistemas de gestión ambiental como una herramienta para lograr el equilibrio entre los aspectos sociales, ambientales y económicos de las instituciones, e incidir en la formación académica de los futuros profesionistas (Aleixo, Azeiteiro y Leal, 2018; Collins, 2017; Mushtaq, Bandh y Shafi, 2020).

Para reconocer las características de las prácticas de gestión ambiental de las instituciones educativas es de utilidad la clasificación de Gudynas (2009): a) la sustentabilidad débil acepta modificar los procesos productivos para reducir el impacto ambiental y considera necesaria la conservación para el crecimiento económico; $b$ ) la sustentabilidad fuerte sostiene que el valor de la naturaleza no puede reducirse a la monetización, y c) la sustentabilidad súper fuerte indica que el ambiente debe ser valorado desde múltiples dimensiones, incluso más importantes que la económica.

A principios del siglo XXI algunas universidades de Estados Unidos de América dieron los primeros pasos en la búsqueda de la sustentabilidad, entre ellas Harvard, Stanford y Búfalo. En España, las universidades de Murcia, Vigo, Alicante y Madrid se sumaron a 


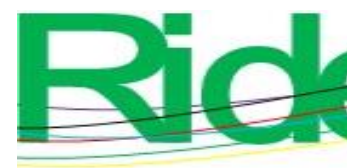

\section{Revista Iberoamericana para la Investigación y el Desarrollo Educativo ISSN 2007 - 7467}

esta oleada (Rivas, 2011); España también impulsó las ecoescuelas en el nivel básico (Trukšāns, 2017). Mientras que en América Latina, Chile y Cuba promovieron programas de gestión ambiental en centros escolares (Ministerio del Medio Ambiente, 2020; Ochoa, Gallardo, Pérez y Ávila, 2016).

México muestra avances en este rubro. Uno de los más destacados fue la creación del Consorcio Mexicano de Programas Ambientales Universitarios para el Desarrollo Sustentable [Complexus] (2020), institución que se centra en la mejora de los procesos académicos para integrar la gestión ambiental y el desarrollo sustentable en los programas de estudio. En educación básica, el Programa Escuela Sustentable promueve estrategias para sistematizar prácticas de gestión ambiental. De 2011 a 2013 se certificaron 1269 escuelas en el ciclo 2018-2019 (Secretaría de Medio Ambiente y Recursos Naturales [Semarnat], 30 de octubre de 2019).

Cabe mencionar que la evaluación de las prácticas de gestión ambiental que se desarrollan en este tipo programas se circunscribe a la aplicación de listas de verificación para comprobar el cumplimiento de indicadores de organización o infraestructura; y la metodología de los estudios que los documentan suele ser cuantitativa (Lo-Iacono, Torregrosa y Capuz, 2017; Testa, Iraldo y Daddi, 2017). En el ámbito educativo se precisa la incorporación de otro tipo de estrategias para conocer el impacto que tienen sobre los individuos y la comunidad que conforman, sobre todo si se considera el enfoque sistémico de la gestión ambiental, el cual otorga especial importancia a la forma en que se integran las partes: conforma un todo interrelacionado, con un enriquecimiento recíproco derivado de esa retroalimentación continua (Edwards, 2019; Forrest, 2018). Este acercamiento puede realizarse a través del estudio de representaciones sociales.

La teoría y metodología de las representaciones sociales, como señala Mireles (2019), permiten conocer los significados que los actores otorgan a distintos aspectos de la vida escolar. Esta teoría ha sido de utilidad para conocer el papel de la educación ambiental en la formación de niños y jóvenes (Allen y Lalonde, 2020; Calixto, 2019; Ponte y Caballero, 2012; Terrón, 2012; Terrón y González, 2012). Asimismo, a través de las representaciones sociales es posible conocer otros aspectos que involucran al resto de la comunidad escolar, como las ideas, saberes o creencias que se construyen dentro de ella y sus respectivas implicaciones (Cuevas, 2016; Kanyimba, Richter y Raath, 2013). 


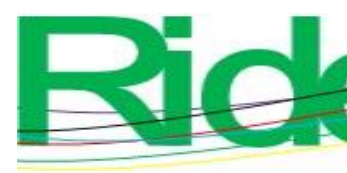

Revista Iberoamericana para la
Investigación y el Desarrollo Educativo
ISSN $2007-7467$

Este tipo de estudios demuestran la fuerte influencia de los medios de comunicación masiva en la integración de las representaciones sociales (Cluley y Green, 2019; Kay y Gaymard, 2020; de Rosa, Dryjanska y Bocci, 2017), ya que influyen en la manera de incorporar nuevos constructos en la vida cotidiana, asignándoles un significado y convirtiéndolas en un producto sociocultural compartido (Ibañez, 2001).

Existen diferentes definiciones de las representaciones sociales; todas tienen en común aspectos que Moscovici (1979) plasma en su definición inicial de 1961:

Una modalidad particular del conocimiento cuya función es la elaboración de los comportamientos y la comunicación entre los individuos. (...) El pasaje del plano de la ciencia al de las representaciones sociales implica una discontinuidad. (...) La ruptura es la condición necesaria para que cada conocimiento físico, biológico, psicológico entre en el laboratorio de la sociedad. Allí todos los conocimientos aparecen, dotados de un nuevo estatuto epistemológico, en forma de representaciones sociales (p. 17).

Jodelet (1986) las categoriza como sistemas de referencia que dan sentido a la información recibida por el individuo en un contexto compartido con otros; también afirma que son dinámicas y se transforman debido a que los grupos sociales modifican constantemente los significados, creando nuevas representaciones.

En cuanto a estructura, la representación social es una unidad funcional con contenido heterogéneo y múltiple, aparentemente disuelto y desordenado, pero que en realidad guarda un orden lógico, en el que pueden distinguirse tres dimensiones: actitudes, información y campo de representación (Moscovici, 1979).

En los estudios de las representaciones sociales predomina una metodología cualitativa (enfoque procesual); se utiliza el método de análisis de contenido para identificar la subjetividad de las expresiones y, dentro de ellas, el sentido y significado que revelan. Como parte de este método, la técnica de analogía y similitud permite categorizar y clasificar la información para definir campos específicos de acuerdo con los sentidos que se atribuyen a ellas (Terrón y González, 2012).

También se usan métodos cuantitativos (enfoque estructural), como la asociación libre de palabras, donde la técnica de redes semánticas naturales permite conocer la forma en que los individuos construyen significados, recuperando sus conocimientos y relacionándolos con la palabra estímulo (Mireles, 2019). Otro método cuantitativo consiste 


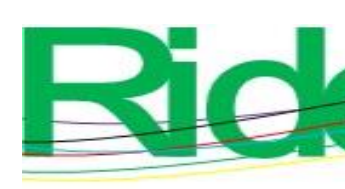

Revista Iberoamericana para la
Investigación y el Desarrollo Educativo ISSN 2007-7467

en preguntas cerradas, cuyas respuestas se pueden procesar con estadística descriptiva. La combinación de ambos en un método mixto es una alternativa para buscar una visión más amplia y sólida, encontrar una mejor comprensión del fenómeno de estudio y obtener resultados más robustos (Abric, 2001; Hernández y Mendoza, 2018).

De acuerdo con este marco teórico-metodológico, es posible considerar que el estudio de las representaciones sociales puede contribuir a revelar las imágenes, valores y actitudes que una comunidad escolar relaciona con la gestión ambiental, y su influencia en la integración de un sistema dirigido al alcance de la sustentabilidad (Yucedag, Kaya y Cetin, 2018). También ayuda a indagar los factores que inciden en la motivación de los involucrados, así como la forma en que asumen su papel en el diseño y ejecución de las tareas, datos que podrían contribuir a explicar las razones por las cuales algunos de estos sistemas no han logrado alcanzar sus objetivos de manera permanente (Benayas, Marcén, Alba y Gutiérrez, 2017).

Si bien el sistema ISO 14001 es uno de los más exitosos en el mundo (Lan y Lee, 2019; Loste, Roldán, Lomba y Giner, 2019), no propone una forma de abordar las funciones sustantivas en las instituciones educativas; se restringe únicamente a actividades técnicas, encaminadas a la remediación (Benayas et al., 2017; Taddei, 2011). Por lo tanto, las investigaciones que se realizan para conocer el alcance de sus objetivos se centran en el análisis cuantitativo de los indicadores, y aunque algunas de ellas abordan aspectos sociales como la comunicación, el liderazgo o el compromiso (Mills et al., 2017; Zhang y Ma, 2021), las conclusiones se obtienen a través de datos estadísticos, los cuales no permiten conocer el pensamiento que construyen los involucrados a través de la información que reciben.

Por ello, se considera que un estudio de la gestión ambiental con una metodología de representaciones sociales puede aportar elementos valiosos para enriquecer su implementación en los centros escolares y favorecer el cumplimiento de los estándares de sustentabilidad. Cabe destacar que, durante la revisión de material bibliográfico, posterior a 2010, no se encontraron antecedentes de trabajos que abordaran la gestión ambiental desde el estudio de las representaciones sociales.

La gestión ambiental para la sustentabilidad promueve la sistematización y divulgación de diversas prácticas, pero también invita a la reflexión crítica que hace posible el despertar de la conciencia y el compromiso para actuar de forma organizada en soluciones efectivas y acordes al contexto (Maldonado y Loza, 2018). 


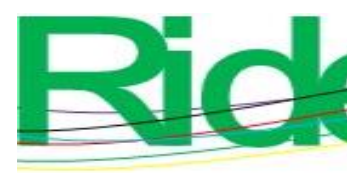

Revista Iberoamericana para la Investigación y el Desarrollo Educativo ISSN 2007 - 7467

La universidad cuenta con antecedentes sobre incorporación de la sustentabilidad en el campus; sin embargo, las iniciativas han sido intermitentes, sin resultados sólidos ni cambios permanentes. Así, se consideró conveniente partir de un estudio que permitiera conocer las representaciones sociales de los miembros, información que contribuirá a determinar los factores que obstaculizan la movilización consciente hacia la gestión ambiental participativa y la sustentabilidad.

\section{Tipo de investigación y alcance}

La investigación fue mixta porque la recolección, análisis e integración de datos se realizó con métodos cuantitativos y cualitativos. El método cuantitativo permitió medir y obtener magnitudes del fenómeno estudiado, las cuales se procesaron mediante procedimientos estadísticos estandarizados. El método cualitativo, por otra parte, permitió acceder a las perspectivas y puntos de vista de los participantes, mediante la observación, análisis e interpretación de los discursos. El diseño de la investigación fue no experimental, de triangulación concurrente, ya que, para corroborar resultados y efectuar una validación cruzada, se aplicaron en paralelo ambos métodos. El alcance fue descriptivo porque se especificaron las características del fenómeno estudiado y se expusieron con sustento teórico y empírico las causas que lo originaron. Su desarrollo comprendió el periodo julio 2019febrero 2020.

Para la aplicación del cuestionario se optó por el censo; se incluyeron preguntas abiertas, de opción múltiple y asociación libre de palabras. En el caso de la entrevista, al ser una técnica cualitativa que no pretende generalizar de manera probabilística los resultados, sino acceder a la interpretación particular de la realidad que construyen los individuos, se optó por elegir a conveniencia representantes de las distintas áreas y niveles educativos. La información obtenida permitió comprender el objeto de estudio en una dimensión amplia.

Se hizo la presentación del proyecto de investigación a representantes de los sectores que integran la comunidad educativa (dirección, alumnado, equipo docente y personal no académico), y se les solicitó apoyo para realizar las actividades en las distintas etapas que lo conforman. Se recibió una respuesta positiva.

La investigación observó los criterios éticos aplicables en humanos establecidos en la Declaración de Helsinki II, la cual fue aprobada por el Comité de Bioética de la Universidad Autónoma de Guerrero (CB-001/2019). Los sujetos de la investigación 


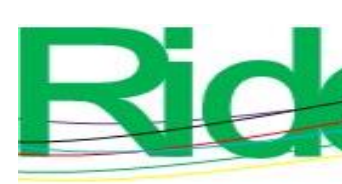

Revista Iberoamericana para la Investigación y el Desarrollo Educativo ISSN $2007-7467$

aceptaron el consentimiento informado, cuyas especificaciones fueron escritas en el cuestionario y expresadas verbalmente en la entrevista. La confidencialidad de los datos se protegió mediante claves asignadas a los participantes para respetar el anonimato.

\section{Procedimiento}

La investigación se realizó en tres fases: diseño de instrumentos, aplicación de instrumentos y análisis y redacción de resultados.

Para la primera fase, después de una revisión bibliográfica, se diseñaron un cuestionario y cuatro entrevistas; el primero permitió acceder a la estructura y las segundas, al contenido de las representaciones sociales. La integración de los dos enfoques (estructural y procesual) ofreció una visión más amplia de la temática abordada. Los instrumentos fueron validados por este grupo de autores, especializados en representaciones sociales, gestión y ciencias ambientales. Se realizó una prueba piloto que consistió en aplicar los instrumentos a un individuo de cada sector de la comunidad escolar (dirección, alumnado, equipo docente y personal no académico) y nivel educativo (secundaria, preparatoria y universidad) para verificar la comprensión del planteamiento de las preguntas, tiempo de aplicación y eficacia de los métodos para el procesamiento de los datos. Después de los ajustes correspondientes y una segunda revisión, se procedió a su aplicación formal.

La aplicación del cuestionario alcanzó un rango amplio de representatividad al tener respuestas significativas en cada sección. Así, se generó una muestra de 278 participantes, lo que permitió obtener un valor de $95 \%$ de confianza y $5 \%$ de error en los datos obtenidos (tabla 1).

Tabla 1. Población y muestra

\begin{tabular}{|l|l|l|l|}
\hline Sector & Población & Respuesta & Porcentaje \\
\hline Directivos & 3 & 3 & $100 \%$ \\
\hline Personal no académico & 17 & 17 & $100 \%$ \\
\hline Profesores & 39 & 37 & $94 \%$ \\
\hline Alumnos & 381 & 221 & $58 \%$ \\
\hline Totales & 440 & 278 & $63 \%$ \\
\hline
\end{tabular}

Fuente: Elaboración propia 


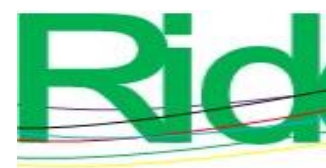

e

El cuestionario se estructuró en cinco secciones (tabla 2).

La asociación libre de palabras permitió identificar elementos que podrían no expresarse verbalmente y estar ocultos en las producciones discursivas. Además, facilitó el acceso al núcleo figurativo de la representación, al expresar en una palabra la imagen o idea evocada con el estímulo, en este caso, gestión ambiental o sustentabilidad.

Tabla 2. Secciones y ejes de análisis del cuestionario

\begin{tabular}{|c|c|c|}
\hline Sección & Ejes de análisis & Tipo de reactivo \\
\hline 1) Datos generales & $\begin{array}{l}\text { Edad, origen, estado civil, género, } \\
\text { escolaridad. }\end{array}$ & Opción múltiple \\
\hline $\begin{array}{l}\text { 2) Fuentes de } \\
\text { información/Conocimiento }\end{array}$ & $\begin{array}{l}\text { Fuentes de información académica. } \\
\text { Tipo de representaciones sociales } \\
\text { que construye con la información } \\
\text { (de la gestión ambiental: reducidas, } \\
\text { globalizadoras, antropocéntrico- } \\
\text { técnicas, integrales o críticas; de la } \\
\text { sustentabilidad: débiles, fuertes y } \\
\text { súper fuertes). }\end{array}$ & $\begin{array}{l}\text { Preguntas } \\
\text { abiertas }\end{array}$ \\
\hline 3) Campo de representación & $\begin{array}{l}\text { Palabras que relaciona con la gestión } \\
\text { ambiental y la sustentabilidad. }\end{array}$ & $\begin{array}{l}\text { Asociación libre } \\
\text { de palabras }\end{array}$ \\
\hline 4) Finalidad/Enfoque & $\begin{array}{l}\text { Meta que se persigue con la gestión } \\
\text { ambiental. } \\
\text { Dimensión de la sustentabilidad con } \\
\text { la que se identifica a la gestión } \\
\text { ambiental. }\end{array}$ & $\begin{array}{l}\text { Valoración } \\
\text { numérica escalar } \\
\text { otorgada a los } \\
\text { ítems }\end{array}$ \\
\hline 5) Estrategias/Actividades & $\begin{array}{l}\text { Frecuencia de actividades o } \\
\text { estrategias relacionadas con la } \\
\text { gestión ambiental que realiza la } \\
\text { institución. }\end{array}$ & Escala Likert \\
\hline
\end{tabular}




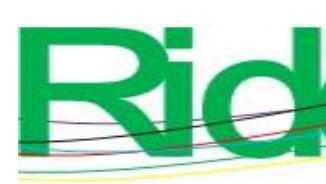

Revista Iberoamericana para la Investigación y el Desarrollo Educativo ISSN 2007 - 7467

\begin{tabular}{|l|l|l|}
\hline & $\begin{array}{l}\text { Frecuencia de la participación } \\
\text { individual y colectiva en dichas } \\
\text { actividades. }\end{array}$ & \\
\hline 6) Actitudes/Participación & $\begin{array}{l}\text { Tendencias hacia la participación en } \\
\text { un programa de gestión ambiental. }\end{array}$ & $\begin{array}{l}\text { Preguntas } \\
\text { abiertas }\end{array}$ \\
& $\begin{array}{l}\text { Tipo de actitudes que se manifiestan } \\
\text { ante la posibilidad de implementar } \\
\text { un programa de gestión ambiental. }\end{array}$ & \\
\hline
\end{tabular}

Nota: El cuestionario puede consultarse en https://forms.gle/c7mpGwjiwMv5YRb79.

Fuente: Elaboración propia

Se diseñaron cuatro entrevistas, una para cada sector: dirección, equipo docente, alumnado y personal no académico. Las preguntas se organizaron en cinco categorías con sus ejes de análisis. Esta herramienta permitió acceder al contexto particular del entrevistado, su bagaje cultural y los argumentos que apoyan su postura y revelan los significados construidos respecto al objeto de estudio (tabla 3 ).

Tabla 3. Categorías y ejes de análisis de las entrevistas

\begin{tabular}{|l|l|}
\hline \multicolumn{1}{|c|}{ Categorías } & \multicolumn{1}{c|}{ Ejes de análisis } \\
\hline 1) Sentido y significado & Saberes, ideas, valores y relaciones \\
\hline 2) Función y finalidad & Vinculación con la labor que realiza \\
& Integración \\
Acciones
\end{tabular}

Fuente: Elaboración propia 

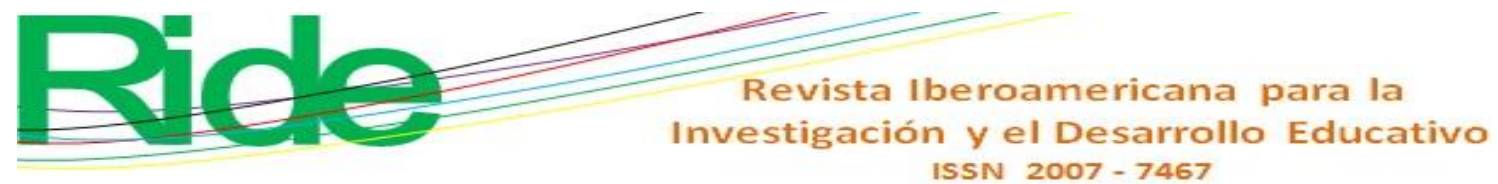

Durante la segunda fase se aplicó el cuestionario a través de un formulario de Google; ofreció la ventaja de concentrar la información vía electrónica y facilitar el procesamiento de datos de manera rápida y eficiente. Fue enviado por correo electrónico a todos los miembros de la comunidad escolar. La población y muestra se detallan en la tabla 1.

Se aplicaron 11 entrevistas: al director general; tres maestros, uno por cada sección (secundaria, preparatoria y universidad); dos representantes del personal no académico (recepción y mantenimiento) y cinco estudiantes (dos de secundaria, dos de preparatoria y uno de universidad).

En la tercera fase se analizaron las respuestas a las preguntas abiertas del cuestionario y las entrevistas con el método de análisis de contenido y la técnica de analogía y similitud. La información obtenida de la asociación libre de palabras se sistematizó con la técnica de redes semánticas naturales; las respuestas del cuestionario se procesaron con estadística descriptiva.

\section{Resultados}

Se analizan los resultados en dos subapartados, identificando las respuestas de cuestionarios y entrevistas; ambos se fortalecen con algunos testimonios.

\section{Representaciones sociales de la gestión ambiental para la sustentabilidad (cuestionarios)}

Se encontraron datos heterogéneos en edad y escolaridad, dado que se consideró a toda la comunidad del centro educativo. Respecto a las otras variables, predominó la población nacida en Acapulco. La mayoría de los participantes son solteros, ya que es población infantil y juvenil en edad escolar. Se encontró un equilibrio entre la participación de hombres y mujeres, como se muestra a continuación:

- $\quad$ Edad: $11-21$ años $=74 \% ; 22-32=13 \% ; 33-43=5 \% ; 44-63=8 \%$.

- Origen: Acapulco de Juárez $=74 \%$; otros municipios del mismo estado = $8 \%$; otros lugares del país o del extranjero $=18 \%$.

- $\quad$ Estado civil: solteros $=87 \%$; casados $=11 \%$; divorciados $=2 \%$.

- Género: hombres $=50 \%$; mujeres $=50 \%$. 


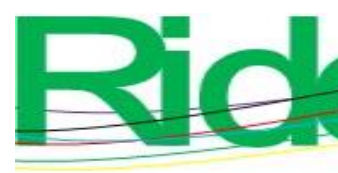

Revista Iberoamericana para la
Investigación y el Desarrollo Educativo
ISSN $2007-7467$

- $\quad$ Escolaridad: primaria $=1 \%$; secundaria $=27 \%$; preparatoria $=42 \%$; licenciatura $=24 \% ;$ maestría $=5 \%$; doctorado $=1 \%$.

La información que ha recibido la comunidad educativa acerca del cuidado de los recursos naturales o el manejo de residuos sólidos a lo largo de su formación académica provino de asignaturas relacionadas con las ciencias naturales, principalmente la biología (26.7\%). Áreas como la formación cívica (6.26\%) y la ética (1.4\%) fueron poco mencionadas. Turismo sustentable $(0.28 \%)$, economía $(0.28 \%)$ y desarrollo sustentable $(0.28 \%)$ tuvieron una sola mención.

Acerca del significado que le otorgan al término gestión ambiental:

- $\quad 41 \%$ aludió a representaciones sociales reducidas, caracterizadas por el enfoque naturalista, con énfasis en el cuidado ambiental. "Es cuando hacemos acciones para cuidar el medio ambiente" (ASM85).

- $\quad 30 \%$ manifestó desconocer o no estar informada acerca de la gestión ambiental.

- $14 \%$ asoció las representaciones sociales antropocéntrico-técnicas, que identifican a la gestión ambiental con un proceso administrativo de los recursos naturales. "Es una forma de organizar y administrar los recursos naturales, al igual que establecer normas para su cuidado" (APM168).

- $\quad 7 \%$ dio otras respuestas. "Promueve los problemas ambientales" (APH165).

- $4 \%$ refirió representaciones globalizadoras, que aluden a una relación armónica con la naturaleza, pero sin integrar apropiadamente lo social. "La gestión ambiental se trata de hacer planes para que el daño al medio ambiente disminuya lo más que se pueda para así poder obtener un ambiente sano" (ASH45).

- $4 \%$ aludió a representaciones integrales, que incorporan aspectos físicos y sociales desde una perspectiva humanista. "Implica estrategias que se deben organizar, actividades para conseguir una calidad de vida mejor donde podemos desarrollar alguna gestión para prevenir o minimizar los casos de contaminación” (PUM255).

- $\quad$ No se encontraron representaciones críticas.

En lo concerniente al campo de las representaciones de la sustentabilidad, $31 \%$ de las personas desconoce el significado del concepto. Además, $30 \%$ se inclinó por la sustentabilidad débil, pues la relacionan con la conservación de los recursos naturales. "Es 

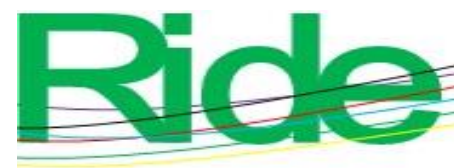

Revista Iberoamericana para la Investigación y el Desarrollo Educativo ISSN 2007 - 7467

mantener o preservar los elementos de la naturaleza, para que las generaciones futuras también puedan disfrutar de estos" (APM81).

Las representaciones sociales de la sustentabilidad fuerte, que valoran la importancia de utilizar los recursos naturales reconociendo su valor intrínseco, alcanzaron $14 \%$. "Permitir que el ambiente tenga los recursos necesarios para seguir existiendo y se sustente así la vida que en él existe” (PLM227).

La sustentabilidad súper fuerte, que pugna por una nueva ética enfocada en el biocentrismo, la valoración múltiple de los recursos, así como la justicia social y ecológica, registró $1 \%$. "Saber cuidar la vida, tratar de reutilizar algo, no comprar algo innecesario para así poder mejorar el medio ambiente y que todos los seres humanos estén bien” (ASM3).

Los componentes del campo de representación se obtuvieron de la asociación libre de palabras. La figura 1 muestra que reciclar, reutilizar, ambiente y naturaleza integran el centro de la red semántica; las 11 palabras restantes, aunque mantienen relación con ellas, no tienen un peso significativo. Para la comunidad escolar, la gestión ambiental se objetiva en reciclar y reutilizar, ya que son los términos que obtuvieron el mayor peso semántico.

El orden y la configuración del núcleo central que se observa en la figura 1 muestra que las personas relacionan la gestión ambiental con actividades de remediación y el enfoque que se les brinda es el de cuidado del ambiente físico-natural, únicamente. 


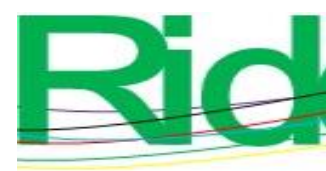

Revista Iberoamericana para la Investigación y el Desarrollo Educativo ISSN $2007-7467$

Figura 1. Núcleo central de las representaciones sociales de la gestión ambiental

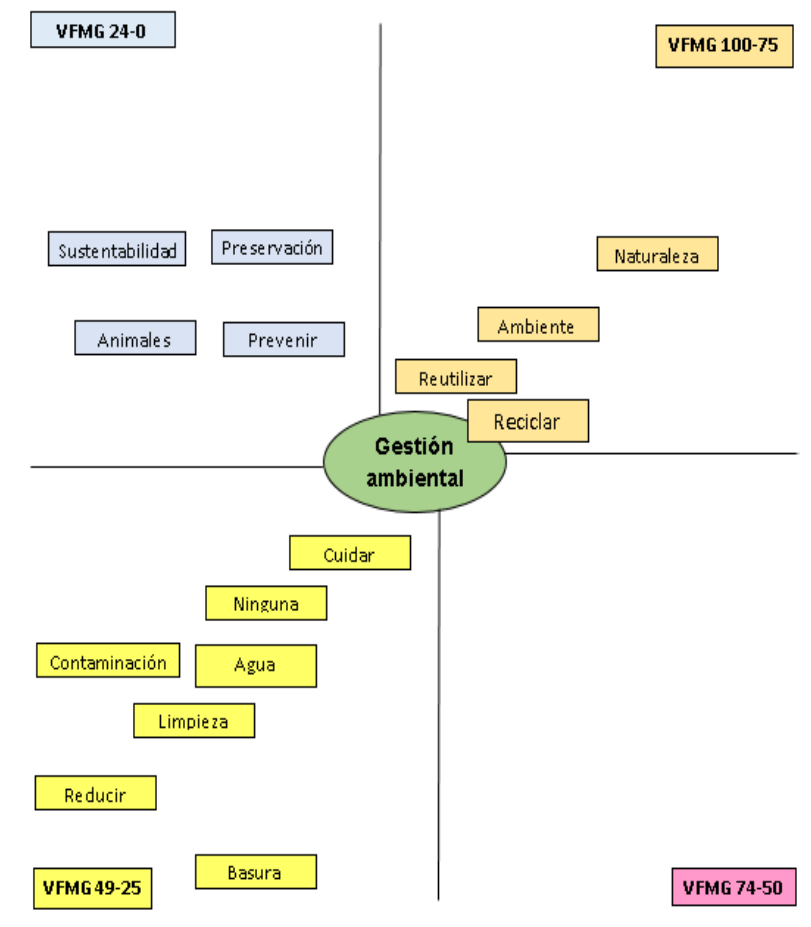

Nota: El esquema tiene cuatro cuadrantes, al centro está el objeto de representación. Cada cuadrante representa $25 \%$ de la distancia semántica entre las palabras. Se lee en el sentido de las manecillas del reloj. Los cuadrantes están ordenados de mayor a menor peso semántico y cada palabra se acerca o se aleja del centro de acuerdo con el peso obtenido.

Fuente: Mireles (2019)

Para $47 \%$ de los encuestados, la finalidad de la gestión ambiental es ahorrar, administrar los recursos y cumplir con la normativa, por lo que el enfoque que se le atribuye es principalmente económico.

Respecto a la sustentabilidad en campus, las estrategias o actividades que los miembros de la comunidad escolar reconocen como frecuentes son el cuidado de áreas verdes (67 \%) y el ahorro de energía (60 \%). Las que no se realizan son la detección de fugas de agua (30 \%) y el uso de energías alternativas (9\%). En docencia destacan las estrategias que promueven una convivencia sana, igualdad de derechos y equidad (36\%); seguidas por eventos en los que se menciona el cuidado del ambiente (35\%) y trabajos de investigación, con propuestas para el aprovechamiento adecuado de recursos (30\%). Las actividades que menos se realizan (5\%) son campañas sobre cuidado del agua o la energía y conferencias sobre consumo responsable, hábitos alimenticios o manejo de residuos sólidos. 


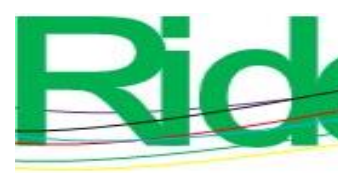

Revista Iberoamericana para la Investigación y el Desarrollo Educativo ISSN $2007-7467$

Sobre las actitudes y participación en programas de gestión ambiental, la respuesta de $80 \%$ de los encuestados fue positiva; manifestaron expresiones como la siguiente: "Creo que todo el personal de la escuela apoyaría la causa y aportarían de su parte para poder realizar con éxito la campaña” (APM8).

Aunado a ello, $16 \%$ ofreció respuestas diversas (necesidad de hacer encuestas y fomentar los valores en el hogar); $3 \%$ manifestó no saber, y menos de $1 \%$ expresó que podría haber respuesta negativa debido a la apatía o la carga extra de trabajo que significaría. Al principio creo que los alumnos se negarían ya que son muy perezosos para hacer trabajo extra (...), también creo que se negarían algunos padres al participar en esto, ya que no querrían aportar algo económicamente pero después de eso no creo que haya más reacciones negativas (ASM19).

\section{Representaciones sociales de la gestión ambiental para la sustentabilidad}

\section{(entrevistas)}

Acerca del sentido y significado, para los entrevistados (dirección, equipo docente, alumnado y personal no académico) la gestión ambiental está dirigida a mejorar el impacto en el ambiente, contribuir, cuidar, restituir el daño causado y minimizar el daño climático (50\%); incluyeron comentarios con valoraciones de la responsabilidad de los humanos en las afectaciones causadas al planeta y expresaron que se debe actuar en consecuencia. También manifestaron preocupación por la intensidad del daño ambiental (25\%) y la necesidad de disminuir la contaminación, manteniendo las calles sin basura (12\%). Se mencionó a la investigación como una estrategia de la gestión ambiental para enseñar a los estudiantes a vincular sus conocimientos con los problemas ambientales y buscar soluciones $(13 \%)$.

En relación con la función y finalidad, los directivos y maestros tienen conocimiento sobre cómo integrar la gestión ambiental en sus funciones, ya que lo asocian con la labor académica a través de campañas y proyectos ambientales que se realizan para minimizar el impacto de la actividad humana y cuidar el planeta. El personal administrativo y de mantenimiento no relaciona la gestión ambiental con sus funciones, solo recibe indicaciones y las ejecuta; sin embargo, todos coinciden en que estas actividades fomentan los valores, principalmente la responsabilidad y el respeto. 

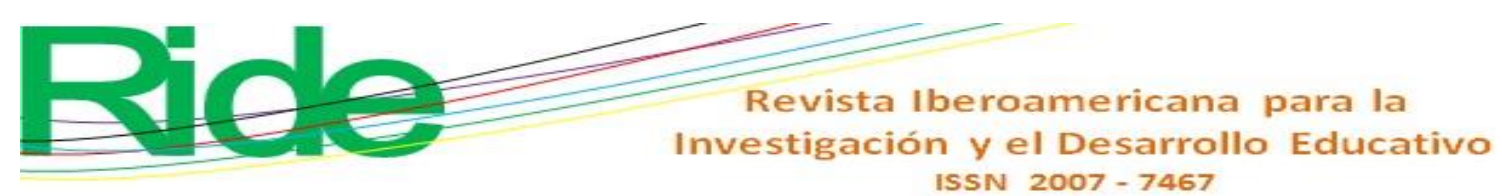

En lo tocante a las fuentes de información, los integrantes de la comunidad escolar se enteran de las actividades de gestión ambiental por las redes sociales e Internet. Los libros de texto que utilizaron en la escuela son la única referencia bibliográfica que les aportó conocimientos sobre estrategias de gestión ambiental. Al interior de la institución, la información se socializa por medio de comentarios, reuniones y campañas; y al exterior, a través de eventos, clubes e información que comparten con la familia y la comunidad.

Sobre las condiciones internas (personales) y externas (institucionales) se identificó que la Dirección General regula las actividades de gestión ambiental de la institución. Algunos maestros las realizan porque son parte de la labor docente, y no por iniciativa propia; sin embargo, expresan que colaboran con entusiasmo. Fuera de la escuela algunos procuran diseñar sus propias estrategias, como se evidencia en los siguientes fragmentos de entrevista.

Tengo que confesar que sí tengo iniciativa propia, no expuesta a la escuela, no a desarrollarla en la escuela, pero sí, a título personal sí soy de las personas que se preocupa y que se ocupa por hacer un poquito de ayuda para esto para que esté mejor (NAF2; 92-95).

Surge por las indicaciones de la Dirección y ya el entusiasmo que le pone uno es por la iniciativa (NAM1; 64-65).

Las representaciones sociales de la gestión ambiental que se identificaron en las respuestas de los entrevistados coinciden con los resultados obtenidos en el cuestionario (reducidas o simples, globalizadoras y antropocéntrico-técnicas), ya que se refieren al deterioro ambiental, así como a medidas de cuidado, mejora y conservación; también se mencionaron la deforestación y la contaminación. Se plantean algunas estrategias para restablecer el equilibrio entre sociedad y naturaleza, así como la necesidad de tomar conciencia para resarcir el daño causado al planeta. Las representaciones antropocéntricotécnicas tienen menor incidencia, pero se reconocen cuando se menciona la administración de los recursos naturales como un mecanismo de conservación que permita seguir aprovechándolos.

Las representaciones sociales de la sustentabilidad también coinciden con las obtenidas en el cuestionario (sustentabilidad débil), ya que los entrevistados refieren actividades como el cuidado de los recursos naturales o la separación y reciclaje de basura, 


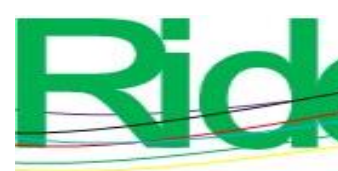

Revista Iberoamericana para la Investigación y el Desarrollo Educativo ISSN $2007-7467$

enfocándose en la esfera económica, al considerar que la administración de los recursos permitirá seguir otorgando al ambiente un carácter utilitario para el ser humano.

\section{Discusión}

El objetivo de esta investigación fue identificar las representaciones sociales sobre la gestión ambiental que comparten los miembros de un centro escolar privado. A continuación, se discuten los principales hallazgos.

Conocer la forma en que la comunidad escolar construye su propia representación social acerca de la gestión ambiental otorga elementos para comprender el dinamismo de sus prácticas, pues cuando los miembros de un grupo asignan un significado a los objetos de representación que hacen comprensible la realidad, este orienta y caracteriza las acciones de las personas (Abric, 2001; Moscovici, 1979).

El sentido que otorga la comunidad escolar de la Universidad Español del campus Diamante a sus representaciones sociales se centra en el cómo y el para qué. Los miembros de la comunidad escolar hacen mención del reciclaje y la reutilización como estrategias para contribuir al cuidado del medio ambiente, y las consideran importantes para la preservación de los recursos para las generaciones futuras. En un estudio realizado sobre representaciones sociales de las prácticas del reciclaje, Ponte y Caballero (2012) obtuvieron resultados similares, aun y cuando se circunscribieron solo al manejo de residuos como parte de la gestión ambiental.

Estos resultados también encuentran concordancia con los obtenidos en otros estudios ya referidos sobre representaciones sociales de la educación ambiental (Allen y Lalonde, 2020; Terrón, 2012; Terrón y González, 2012). Puede inferirse que la formación académica que los individuos recibieron a través del sistema educativo ejerce influencia sobre la representación de la gestión ambiental, replicando el enfoque naturalista que permea en la educación ambiental y que no contempla el ámbito social (Terrón y González, 2012). Los libros de texto se mencionan como la fuente de información que reciben en la escuela, lo que explica la relación entre la educación formal y las representaciones sociales para vincular los conocimientos construidos con las prácticas culturales (Calixto, 2019; Terrón, 2012), lo que, a su vez, se refleja en concepciones y prácticas ambientales reducidas.

Por otra parte, la dimensión vinculada con la actitud se muestra como positiva; la población está dispuesta a actuar para mejorar la situación ambiental. No obstante, se apreció 


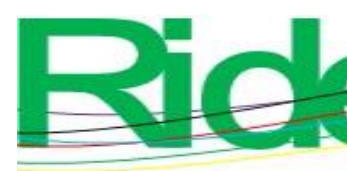

Revista Iberoamericana para la Investigación y el Desarrollo Educativo ISSN $2007-7467$

que las estrategias identificadas por las personas como favorables no logran reflejarse en la práctica dentro de la institución. Lo anterior es relevante porque si bien se considera que una actitud positiva es indispensable para el éxito de los procesos de gestión de proyectos ambientales (Klein et al., 2021; Kitila y Woldemikael, 2019; Mills et al., 2017; Yucedag et al., 2018), también se requiere de representaciones sociales estructuradas, con códigos, valores, lógicas clasificatorias y principios interpretativos suficientemente fuertes para orientar actitudes positivas hacia prácticas que construyan una conciencia colectiva que definan una forma de actuar en el mundo (lbáñez, 2001; Jodelet, 1986; Moscovici, 1979).

En este sentido, los resultados muestran que las representaciones de la gestión ambiental y la sustentabilidad que construyen los sujetos de estudio son heterogéneas, incluso algunos participantes no tienen referencia acerca de estas palabras. Esto sugiere que los miembros de la comunidad escolar no estructuran una representación social con simbolismos y significados compartidos que permita dar sentido a estos términos; por ello, aun cuando las actitudes se manifiesten teóricamente en el deber ser, no lograrán entrar en un campo de representación que permita la organización y apropiación del conocimiento para desencadenar la acción (Ibañez, 2001; de Rosa et al., 2017).

Con esta aportación se da respuesta al carácter episódico de las prácticas ambientales y al poco impacto que resulta de la implementación de la gestión ambiental (Benayas et al., 2017), así como a la carencia de comportamientos que involucren acciones de responsabilidad social y compromiso con el ambiente (Malavé y Fernández, 2020; So et al., 2019). Es importante construir representaciones sociales que otorguen sentido y significado a la gestión ambiental y la sustentabilidad.

Por último, la comunidad escolar identificó el Internet y las redes sociales como las principales fuentes (fuera de la institución) a través de las cuales conocen medidas y estrategias para el cuidado del ambiente. Esta referencia refuerza la información de estudios previos respecto a la fuerte influencia de los medios masivos al tener la facultad de elaborar representaciones y hacerlas circular (Cluley y Green, 2019; Kay y Gaymard, 2020; Moscovici, 1979).

Dentro de los sistemas de gestión ambiental, la comunicación forma parte importante del proceso para la correcta ejecución de las tareas y la difusión de objetivos y resultados (Cluley y Green, 2019; ISO, 2015); sin embargo, no se ha contemplado a los medios de comunicación masiva como una alternativa para acercar la información a los miembros de 


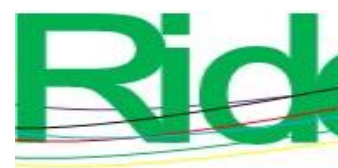

Revista Iberoamericana para la
Investigación y el Desarrollo Educativo
ISSN $2007-7467$

la institución. Tampoco hay evidencias de estudios sobre estrategias o desarrollo de competencias para discriminar el cúmulo de datos que reciben a través de la Red para tomar decisiones más conscientes e informadas, con impacto no solo en el campo laboral sino en la vida cotidiana. Los resultados obtenidos pueden servir como referencia para futuros estudios sobre la influencia de las redes sociales en la construcción de representaciones sociales de la gestión ambiental y la sustentabilidad.

La gestión ambiental como práctica pedagógica que busca contribuir a la sustentabilidad ha evidenciado su valor en estudios que analizan el resultado técnico de la aplicación de proyectos de esta naturaleza (Benayas et al., 2017; Collins, 2017; Rivas, 2001). Sin embargo, no hacen referencia a un sistema de evaluación que mida su impacto en el pensamiento de sentido común que construye la comunidad escolar; por lo que se infiere que, si las representaciones sociales cambian (Moscovici, 1979), una intervención educativa puede lograr la construcción y transformación de sentidos y significados para estructurar con el tiempo representaciones sociales más complejas y dinamizadoras.

\section{Fortalezas y limitaciones}

Como fortaleza, se puede afirmar que los instrumentos diseñados y aplicados en el desarrollo de esta investigación resultaron pertinentes para el objetivo planteado, y son la base para realizar estudios posteriores.

Esta es una investigación transversal, los datos obtenidos corresponden a un momento histórico determinado; por lo tanto, es necesario desarrollar más procesos investigativos para observar la forma en que evolucionan las representaciones sociales de la gestión ambiental. Esto se considera como una limitación. 


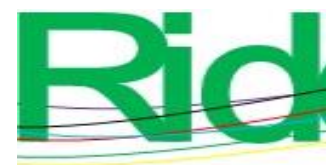

Revista Iberoamericana para la Investigación y el Desarrollo Educativo ISSN $2007-7467$

\section{Conclusiones}

Este trabajo identificó las representaciones sociales sobre la gestión ambiental para la sustentabilidad compartidas por una comunidad escolar. Estas mostraron un fuerte componente conservacionista y utilitario al interior de la institución, relacionado con los sistemas educativos de modelo tradicional.

Asimismo, se constató que los medios de comunicación masiva representan una importante área de oportunidad para la socialización de temas relacionados con la gestión ambiental de la institución en la búsqueda de fortalecer su desempeño, tanto del personal directivo, docente y no académico como del alumnado.

Es necesario desarrollar en la escuela procesos de capacitación y actualización en temas ambientales y de gestión para que toda la comunidad educativa construya representaciones sociales más sólidas, complejas y dinamizadoras en el tránsito hacia un manejo ambiental y sustentable de la institución educativa.

\section{Contribución a futuras líneas de investigación}

Esta investigación genera una oportunidad para impulsar la transformación significativa de los procesos de evaluación de temas ambientales en las instituciones educativas, públicas o privadas: pasar de simples listas de verificación a una verdadera apropiación institucional que conduzca al mejoramiento de los procesos administrativos y educativos y a la promoción de una justicia ambiental con equidad social. 


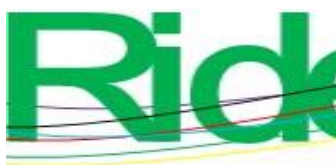
Revista Iberoamericana para la
Investigación y el Desarrollo Educativo
ISSN $2007-7467$

\section{Referencias}

Abric, J. (2001). Metodología de recolección de las representaciones sociales. En Abric, J. (coord.), Prácticas sociales y representaciones. Ciudad de México, México: Ediciones Coyoacán.

Aleixo, A., Azeiteiro, U. and Leal, S. (2017). The implementation of sustainability practices in Portuguese higher education institutions. International Journal of Sustainability in Higher Education, 19(1), 146-178. Retrieved from https://doi.org/10.1108/IJSHE-022017-0016.

Allen, J. and Lalonde, C. (2020). Representations of natural environments, recurring characters, and ways of living with the land in children's retellings of First Nations oral narratives. Early Childhood Research Quarterly, 53, 50-63. Retrieved from https://doi.org/10.1016/j.ecresq.2020.01.005.

Association of University Leaders for a Sustainable Future. (1999). Declaración de líderes de universidades para un futuro sostenible. Declaración de Talloires. Recuperado de http://ulsf.org/wp-content/uploads/2015/06/Spanish_TD.pdf.

Benayas, J., Marcén, C., Alba, D. y Gutiérrez, B. J. (2017). Educación para la Sostenibilidad en España. Reflexiones y propuestas. España: Fundación Alternativas y Red Española para el Desarrollo Sostenible. Recuperado de https://www.fundacionalternativas.org/public/storage/opex_documentos_archivos/8 1ef826c30f2322a5c9c8536a50faf20.pdf.

Calixto, R. (2019). Las representaciones sociales sobre el cambio climático de los estudiantes de pedagogía en México: un acercamiento desde la perspectiva de género. Educación, 28(54), 7-26. Recuperado de https://doi.org/10.18800/educacion.201901.001.

Cluley, R. and Green, W. (2019). Social representations of marketing work: advertising workers and social media. European Journal of Marketing, 53(5), 830-847. Retrieved from https://doi.org/10.1108/EJM-12-2016-0682.

Collins, T. (2017). Review of the twenty-three year evolution of the first university course in green chemistry: teaching future leaders how to create sustainable societies. Journal of Cleaner Production, 140, 93-110. Retrieved from https://doi.org/10.1016/j.jclepro.2015.06.136.

Consorcio Mexicano de Instituciones de Educación Superior para la Sustentabilidad [Complexus]. (2020). ¿Qué es el Complexus? Recuperado de 

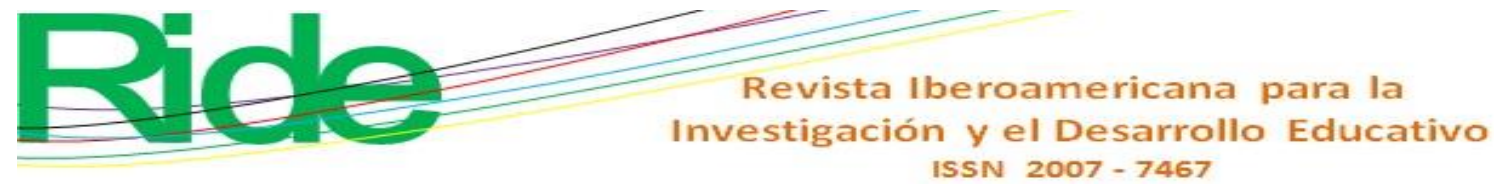

http://complexus.org.mx/\#: :text=El\%20Consorcio\%20toma\%20su\%20nombre,ma yor\%20sin\%20perder\%20su\%20individualidad.

Cuevas, Y. (2016). Recomendaciones para el estudio de representaciones sociales en investigación educativa. Cultura y Representaciones Sociales, 11(21), 109-140. Recuperado de http://www.scielo.org.mx/scielo.php?script=sci_arttext\&pid=S200781102016000200109.

de Andrade, M., Sudan, D., de Meira, A., da Rocha, F. and Sorrentino, M. (2017). Environmental Education in University Management: Contributions to Sustainability Construction at The University of São Paulo, Brazil. In Leal, W., Skanavis, C. do Paço, A., Rogers, J., Kuznetsova, O. and Castro, P. (eds.), Handbook of Theory and Practice of Sustainable Development in Higher Education (pp. 369-385). Cham, Switzerland, Springer. Retrieved from https://doi.org/10.1007/978-3-319-478890_26.

de Rosa, A., Dryjanska, L. and Bocci, E. (2017). Profiling authors based on their participation in academic social networks. Paper presented at the $11^{\text {th }}$ International Technology, Education and Development Conference. Valencia, 6-8 March, 2017. Retrieved from https://www.researchgate.net/publication/339658378_Profiling_authors_based_on_t heir_participation_in_Academic_Social_Networks.

Edwards, D. (2019). Shifting the perspective on community-based management of education: From systems theory to social capital and community empowerment. International Journal of Educational Development, 64, 17-26. Retrieved from https://doi.org/10.1016/j.ijedudev.2018.11.004.

Forrest, J. (2018). General Systems Theory. Cham, Switzerland: Springer. https://link.springer.com/book/10.1007/978-3-030-04558-6

Gudynas, E. (2009). Desarrollo sostenible: posturas contemporáneas y desafíos en la construcción del espacio urbano. Montevideo, Uruguay: Centro Latinoamericano de Ecología Social. $\quad$ Recuperado de http://www.gudynas.com/publicaciones/GudynasDesaSustVPopular09.pdf.

Hernández, R. y Mendoza, C. (2018). Metodología de la investigación. Las rutas cuantitativa, cualitativa y mixta. Ciudad de México, México: McGraw-Hill. 


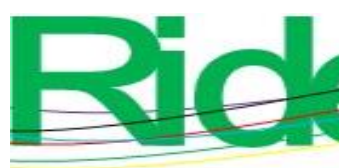

Revista Iberoamericana para la Investigación y el Desarrollo Educativo ISSN 2007 - 7467

Ibañez, T. (2001). Representaciones sociales, teoría y método. En Jiménez, B. (coord.), Psicología social construccionista (2. ${ }^{a}$ ed.) (pp. 153-208). Guadalajara, México: Universidad de Guadalajara.

Jodelet, D. (1986). La representación social: fenómenos, conceptos y teoría. En Moscovici, S. (coord.), Psicología social II (pp. 469-494). Barcelona, España: Paidós.

Kanyimba, A., Richter, B. and Raath, S. (2013). The effectiveness of an environmental management system in selected South African primary schools. Journal of Cleaner Production, 66, 479-488. Retrieved from http://dx.doi.org/10.1016/j.jclepro.2013.10.052.

Kay, N. and Gaymard, S. (2020). Climate change in the Cameroonian press: An analysis of its representations. Public Understanding of Science, 30(4), 417-433. Retrieved from https://doi.org/10.1177/0963662520976013.

Kitila, A. W. and Woldemikael, S. M. (2019). Waste electrical and electronic equipment management in the educational institutions and governmental sector offices of Addis Ababa, Ethiopia. Waste Management, 85, 30-41. Retrieved from https://doi.org/10.1016/j.wasman.2018.12.007.

Klein, L., Schramm, M., Veiga, L. and Moreira, R. (2021). Management of lean waste in a public higher education institution. Journal of Cleaner Production, 286. Retrieved from https://doi.org/10.1016/j.jclepro.2020.125386.

Lan, Y. C. and Lee, S. C. (2019). Development of an Environmental Management System Framework for Hong Kong Higher Education Institutions. In Hu, A., Matsumoto, M., Kuo, T. and Smith, S. (eds.), Technologies and Eco-Innovation towards Sustainability II (pp. 25-38). Singapore: Springer. Retrieved from https://doi.org/10.1007/978-981-13-1196-3_3.

Leff, E. (2008). Saber ambiental. Sustentabilidad, racionalidad, complejidad, poder. México: Siglo XXI Editores.

León, Y., Gomera, A., Antúnez, M., Martínez, B., Villamandos, F. and Vaquero, M. (2017). Enhancing environmental management in universities through participation: the case of the University of Córdoba. Journal of Cleaner Production, 172, 4328-4337. Retrieved from http://dx.doi.org/10.1016/j.jclepro.2017.06.103.

Lo-Iacono, V., Torregrosa, J. y Capuz, S. (2017). Organizational life cycle assessment: suitability for higher education institutions with environmental management systems. 


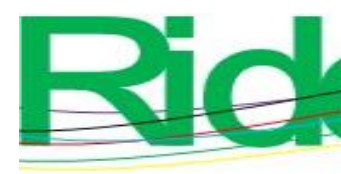

Revista Iberoamericana para la Investigación y el Desarrollo Educativo ISSN $2007-7467$

The International Journal of Life Cycle Assessment, 22, 1928-1943. Retrieved from https://doi.org/10.1007/s11367-017-1289-8.

Loste, N., Roldán, E., Lomba, L. and Giner, B. (2019). Green Chemistry and Environmental Management Systems: Relationships, Synergies, Advantages and Barriers of Joint Implementation at Universities. Environmental Management, 64, 783-793. Retrieved from https://doi.org/10.1007/s00267-019-01218-y.

Malavé, E. y Fernández, M. (2020). Gestión ambiental de las empresas públicas y privadas en la ciudad de Guayaquil - Ecuador y su incidencia en el desarrollo sostenible. Sinergias Educativas, 1(5), 204-223. Recuperado de http://portal.amelica.org/ameli/jatsRepo/382/3821581012/3821581012.pdf.

Maldonado, T. y Loza, O. (2018). Orientaciones para el diseño del programa educativo y social de los centros de educación y cultura ambiental. Ciudad de México, México: Secretaría de Medio Ambiente y Recursos Naturales. Recuperado de https://biblioteca.semarnat.gob.mx/janium/Documentos/Ciga/libros2018/CD003014 .pdf.

Mikulčić, H., Duić, N. and Dewil, R. (2017). Environmental management as a pillar for sustainable development. Journal of Environmental Management, 203, 867-871. Retrieved from https://doi.org/10.1016/j.jenvman.2017.09.040.

Mills, J., Gaskell, P., Ingram, J., Dwyer, J., Reed, M. and Short. C. (2017). Engaging farmers in environmental management through a better understanding of behavior. Agriculture and Human Values, 34, 283-299. Retrieved from https://doi.org/10.1007/s10460-016-9705-4.

Ministerio del Medio Ambiente. (2020). Manual para la gestión ambiental en establecimientos educacionales. Santiago, Chile: Gobierno de Chile. Recuperado de https://mma.gob.cl/wp-content/uploads/2020/11/Manual-Gestio\%CC\%81nAmbiental-para-EE.pdf.

Mireles, O. (2019). Representación social de la excelencia académica. Un estudio en el posgrado de la UNAM. Ciudad de México, México: Instituto de Investigaciones sobre la Universidad y la Educación.

Moscovici, S. (1979). El psicoanálisis, su imagen y su público (2. ${ }^{\mathrm{a}}$ ed.). Buenos Aires, Argentina: Huemul. 

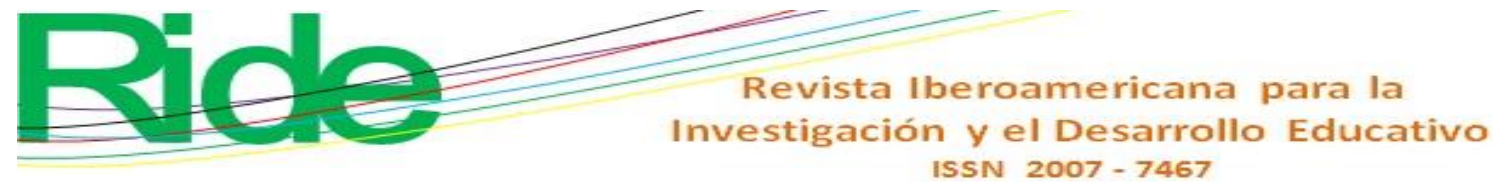

Mushtaq, B., Bandh, S. and Shafi, S. (2020). Environmental Education and Environmental Impact Assessment. In Environmental Management (pp. 95-148). Singapore: Springer. Retrieved from https://doi.org/10.1007/978-981-15-3813-1_3.

Ochoa, M., Gallardo, O., Pérez, R. y Ávila, R. (2016). Tecnología para la gestión ambiental integral en instituciones escolares. Aplicación en Holguín. Ciencias Holguín, 22(1), $1-16$.

Organización Internacional de Normalización [ISO]. (2015). ISO 14001:2015. Recuperado de https://www.nueva-iso-14001.com/pdfs/FDIS-14001.pdf.

Ponte, C. y Caballero, M. (2012). Representaciones sociales de la práctica del reciclaje en el Instituto Pedagógico de Caracas. En Calixto, R. (coord.), En la búsqueda de los sentidos y significados de la educación ambiental (pp. 149-170). Ciudad de México, México: Universidad Pedagógica Nacional.

Rivas, M. (2011). Modelo de sistema de gestión ambiental para formar universidades ambientalmente sostenibles en Colombia. Gestión y Ambiente, 14(1), 151-162. Recuperado https://revistas.unal.edu.co/index.php/gestion/article/view/25453/39275.

Salim, H., Padfield, R., Hansen, S., Mohamad, S., Yuzir, A., Syayuti, K., Tham, M. and Papargyropoulou, E. (2018). Global trends in environmental management system and ISO14001 research. Journal of Cleaner Production, 170, 645-653. Retrieved from https://doi.org/10.1016/j.jclepro.2017.09.017.

Secretaría de Medio Ambiente y Recursos Naturales [Semarnat]. (30 de octubre de 2019). La Semarnat y el Cecadesu promueven el manejo ambiental en escuelas. (Comunicado de prensa). Recuperado de https://www.gob.mx/semarnat/prensa/lasemarnat-y-el-cecadesu-promueven-el-manejo-ambiental-en-escuelas?idiom=es.

So, W., Lee, J. and Chow, C. (2019). Environmental Sustainability and Education for Waste Management. In So, W., Chow, C. and Lee, J. (eds.), Environmental Sustainability and Education for Waste Management (pp. 1-11). Singapore: Springer. Retrieved from https://doi.org/10.1007/978-981-13-9173-6_1.

Taddei, J. (2011). Cómo avanzar hacia la sustentabilidad en las instituciones de educación superior (SGSU). México: Jorale Editores-Universidad de Sonora.

Terrón, E. (2012). Horizontes de la educación ambiental. Un estudio de representaciones sociales. En Calixto, R. (coord.), En la búsqueda de los sentidos y significados de la 


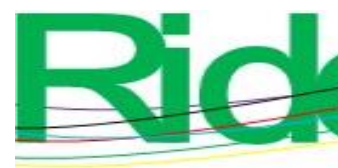

Revista Iberoamericana para la
Investigación y el Desarrollo Educativo
ISSN $2007-7467$

educación ambiental (pp. 129-148). Ciudad de México, México: Universidad Pedagógica Nacional.

Terrón, E. y González, E. (2012). Representaciones sociales de la educación ambiental. Influencia de los discursos ambientales en las representaciones construidas y sus implicaciones educativas. España: Editorial Académica Española.

Testa, F., Iraldo, F. and Daddi, T. (2017). The Effectiveness of EMAS as a Management Tool: A Key Role for the Internalization of Environmental Practices. Organization \& Environment, $\quad 31(1), \quad 48-69 . \quad$ Retrieved from https://doi.org/10.1177/1086026616687609.

Trukšāns, D. (2017). Towards Municipal Environmental Development: Environmental Management Systems for Local Formal Education Establishments. Regional Formation and Development Studies, 23(3), 175-185. Retrieved from https://doi.org/10.15181/rfds.v23i3.1594.

Yucedag, C., Kaya, L. and Cetin, M. (2018). Identifying and assessing environmental awareness of hotel and restaurant employees' attitudes in the Amasra District of Bartin. Environmental Monitoring and Assessment, 190, 1-8. Retrieved from https://doi.org/10.1007/s10661-017-6456-7.

Vilchis, T. E., Aparicio, J. L. y Rodríguez, C. (2021). Representaciones sociales de la gestión ambiental, construcción desde la nueva normalidad. En Educación, innovación y nueva normalidad (pp. 386-395). Durango, México: Asociación Normalista de Docentes Investigadores.

Zhang, Q. and Ma, Y. (2021). The impact of environmental management on firm economic performance: The mediating effect of green innovation and the moderating effect of environmental leadership. Journal of Cleaner Production, 292, 126057. Retrieved from https://doi.org/10.1016/j.jclepro.2021.126057. 


\begin{tabular}{|c|c|}
\hline & $\begin{array}{l}\text { Revista lberoamericana para la } \\
\text { Investigación y el Desarrollo Educativo } \\
\text { ISSN } 2007-7467\end{array}$ \\
\hline Rol de Contribución & Autor (es) \\
\hline Conceptualización & $\begin{array}{l}\text { Trinidad Esmeralda Vilchis Pérez y José Luis Aparicio López } \\
\text { «igual» }\end{array}$ \\
\hline Metodología & $\begin{array}{l}\text { Esperanza Terrón Amigón «principal» y Trinidad Esmeralda } \\
\text { Vilchis Pérez «que apoya» }\end{array}$ \\
\hline Software & Columba Rodríguez Alviso «principal» \\
\hline Validación & $\begin{array}{l}\text { Trinidad Esmeralda Vilchis Pérez y Columba Rodríguez } \\
\text { Alviso «igual» }\end{array}$ \\
\hline Análisis Formal & Hilda Janet Arellano Wences «principal» \\
\hline Investigación & $\begin{array}{l}\text { Trinidad Esmeralda Vilchis Pérez «principal», José Luis } \\
\text { Aparicio López «que apoya» y Columba Rodríguez Alviso } \\
\text { «que apoya», Hilda Janet Arellano Wences «que apoya» }\end{array}$ \\
\hline Recursos & $\begin{array}{l}\text { Trinidad Esmeralda Vilchis Pérez «principal» e Hilda Janet } \\
\text { Arellano Wences «que apoya» }\end{array}$ \\
\hline Curación de datos & $\begin{array}{l}\text { Trinidad Esmeralda Vilchis Pérez «principal» y Rosa María } \\
\text { Brito Carmona «que apoya» }\end{array}$ \\
\hline $\begin{array}{l}\text { Escritura - Preparación del } \\
\text { borrador original }\end{array}$ & Trinidad Esmeralda Vilchis Pérez «principal» \\
\hline $\begin{array}{l}\text { Escritura - Revisión y } \\
\text { edición }\end{array}$ & $\begin{array}{l}\text { José Luis Aparicio López, Columba Rodríguez Alviso y } \\
\text { Esperanza Terrón Amigón «igual» }\end{array}$ \\
\hline Visualización & Columba Rodríguez Alviso «principal» \\
\hline Supervisión & José Luis Aparicio López «principal» \\
\hline Administración de Proyectos & José Luis Aparicio López «principal» \\
\hline Adquisición de fondos & $\begin{array}{l}\text { Trinidad Esmeralda Vilchis Pérez y Columba Rodríguez } \\
\text { Alviso «igual» }\end{array}$ \\
\hline
\end{tabular}

